\title{
Mapping Long-Lived Dark States in Copper
}

\section{Porphyrin Nanostructures}

Hannes Hartmann ${ }^{1}$, Ingo Barke ${ }^{l, *}$, Aleksej Friedrich ${ }^{1}$, Per-Arno Plötz ${ }^{l}$, Olga S. Bokareva ${ }^{1}$, Mohammadreza Bahrami ${ }^{l}$, Kevin Oldenburg $^{l}$, Johannes A. A. W. Elemans ${ }^{2}$, Robert Irsig ${ }^{l}$, KarlHeinz Meiwes-Broer ${ }^{1}$, Oliver Kühn ${ }^{1}$, Stefan Lochbrunner ${ }^{1}$, Sylvia Speller ${ }^{1}$

${ }^{1}$ University of Rostock, Institute for Physics, Albert-Einstein Str. 23, 18059 Rostock

${ }^{2}$ Radboud University Nijmegen, Institute for Molecules and Materials, Heyendaalseweg 135, 6525 AJ Nijmegen

\section{Supporting Information}

\section{S1: DFT calculations of isolated CuTUP}

Density functional theory (DFT) calculations are employed to obtain more insight into the magnitude of the IP and the electronic structure of CuTUP molecules. The calculations are performed for isolated CuTUP in the gas phase, where the undecyl groups have been replaced by hydrogen atoms. In order to provide a reliable description of charge transfer states, the long-range corrected functional LC-BLYP has been used. ${ }^{1,2}$ Here, the exact exchange is switched-on via an error function to provide the correct asymptotic behavior, void of the self-interaction error. The switching rate is controlled by the parameter $\omega$, which can be obtained in a systematic way. Although there are $\omega$-values that are supposed to be valid for whole classes of systems, recent 
investigations have shown that, especially for metal-ligand complexes, system-specific $\omega$ optimization yields values which differ considerably from standard ones. ${ }^{3-5}$ A common strategy for $\omega$-optimization builds on the $\triangle \mathrm{SCF}$ method and as such is well-suited to calculate IPs. ${ }^{6,7}$ Starting point is the observation that in exact Kohn-Sham theory the IP of the $N$ electron system is equal to the negative of the energy of the HOMO orbital. ${ }^{8}$ The IP can be written as

$$
I P(\omega, N)=E_{g s}(\omega, N-1)-E_{g s}(\omega, N)
$$

In view of the limit of the exact Kohn-Sham theory this suggest the definition of the following function to be minimized with respect to the parameter $\omega$

$$
J(\omega)=\left|\varepsilon_{\text {номо }}(\omega, N)+I P(\omega, N)\right| \quad \text { Eq. S1.2 }
$$

where $\varepsilon_{\text {номо }}(\omega, N)$ is the $\omega$-dependent HOMO energy of the $N$ electron system. The improved description of molecular properties such as the IP, provided by the $\omega$-optimized LC-BLYP functional, has been demonstrated in a number of publications (see, e.g., ref. 9).

The electronic structure of the gas phase CuTUP has been modeled using the DFT with the LCBLYP functional and the 6-31G(d) basis set as implemented in Gaussian 09. ${ }^{10}$ First, geometry optimization was performed for the doublet ground state assuming $\mathrm{D}_{4 \mathrm{~h}}$ symmetry. Subsequently, the $J(\omega)$, eq. S1.2, was minimized by scanning on a certain range of $\omega$-values as shown in Fig. S1.1. Thereby, it is assumed that the cation has triplet multiplicity. In fact the ground state of the triplet cation was found to be more stable than that of the respective singlet by $0.36 \mathrm{eV}$. In a next step the optimization was performed starting from the quartet ground state and using the same geometry, see Figure S1. Here, the cation is of triplet multiplicity as well. Its stability with respect to the quintet state was calculated to be $2.11 \mathrm{eV}$. Note that the mentioned orders of stability hold for the range of $\omega$ parameters shown in Figure S1. From Figure S1 we find an optimal $\omega$ of 0.23 for both the doublet and the quartet state. Note that this value is similar to those of Ir-based organometallics used as photosensitizers (ca. 0.18), ${ }^{3}$ but notably different from standard values used in common electronic structure codes. ${ }^{2,11}$ 


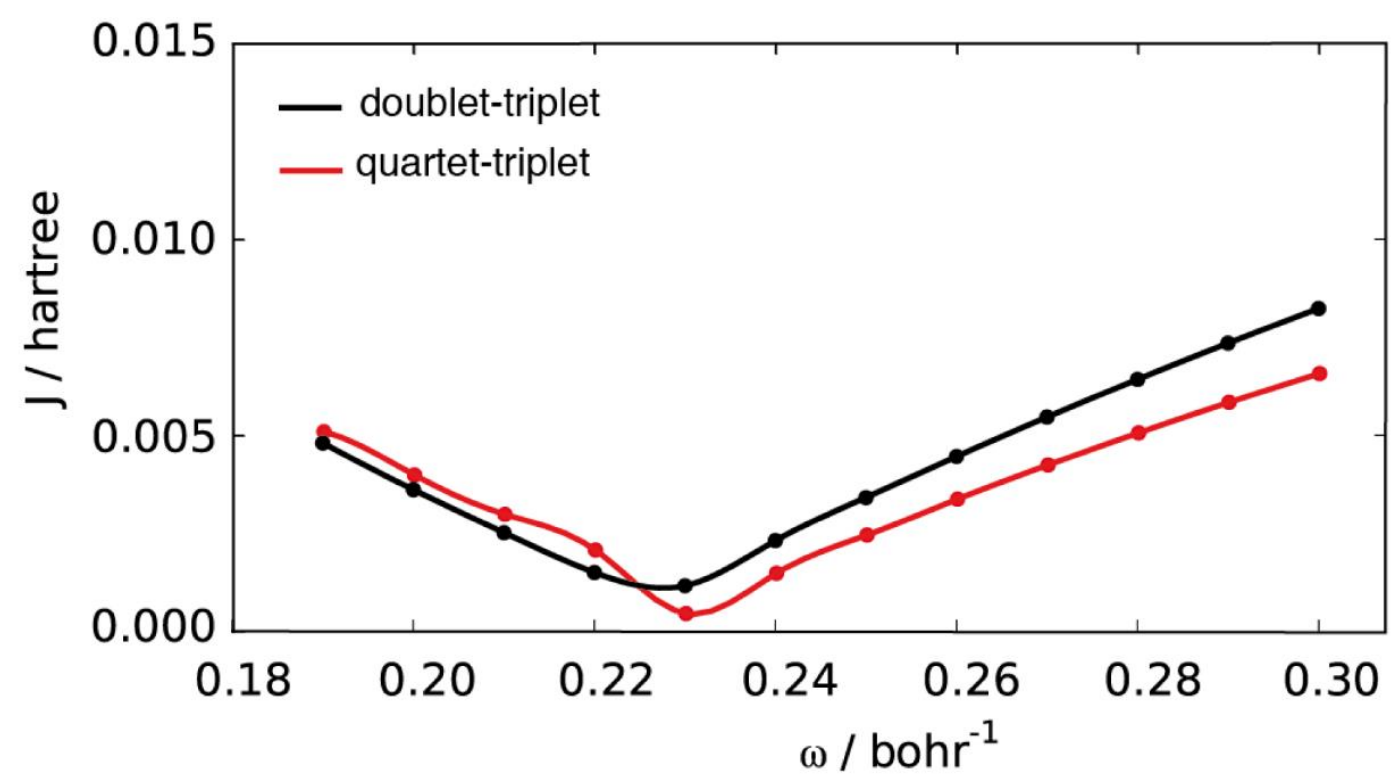

Figure S1. Optimization of $\omega$-parameter for the LC-BLYP functional according to eq. S2.2.

\section{REFERENCES}

(1) Iikura, H.; Tsuneda, T.; Yanai, T.; Hirao, K. A. Long-range correction scheme for generalized-gradient-approximation exchange functionals. J. Chem. Phys. 2001, 115, $3540-3544$.

(2) Tawada, Y.; Tsuneda, T.; Yanagisawa, S.; Yanai, T.; Hirao, K. A long-range-corrected time-dependent density functional theory. J. Chem. Phys. 2004, 120, 8425-8433.

(3) Bokareva, I. S.; Grell, G.; Bokarev, S. I.; Kühn, O. Tuning range-separated density functional theory for photocatalytic water splitting systems. J. Chem. Theory Comput. 2015, $11,1700-1709$.

(4) Bokarev, S. I.; Bokareva, O. S.; Kühn, O. A theoretical perspective on charge transfer in 
photocatalysis. The example of Ir-based systems Coord. Chem. Rev. 2015, 304, 133-145.

(5) Bokareva, O. S.; Kühn, O. Quantum chemical study of the electronic properties of an iridium-based photo-sensitizer bound to medium-sized silver clusters. Chem. Phys. 2015, $457,1-6$.

(6) Livshits, E.; Baer, R. A well-tempered density functional theory of electrons in molecules. Phys. Chem. Chem. Phys. 2007, 9, 2932-2941.

(7) Stein, T.; Kronik, L.; Baer, R. Prediction of charge-transfer excitations in coumarin-based dyes using a range-separated functional tuned from first principles. J. Chem. Phys. 2009, $131,244119$.

(8) Perdew, J.; Levy, M. Comment on "Significance of the highest occupied Kohn-Sham eigenvalue". Phys. Rev. B 1997, 56, 16021-16028.

(9) Gallandi, L.; Marom, N.; Rinke, P.; Körzdörfer, T. Accurate ionization potentials and electron affinities of acceptor molecules II: Non-empirically tuned long-range corrected hybrid functionals. J. Chem. Theory Comput. 2016, 12, 605-614.

(10) Frisch, M. J.; Trucks, G. W.; Schlegel, H. B.; Scuseria, G. E.; Robb, M. A.; Cheeseman, J. R.; Scalmani, G.; Barone, V.; Mennucci, B.; Petersson, G. A.; et al. Gaussian 09, Revision D.01; Gaussian, Inc.: Wallingford, CT, 2009.

(11) Song, J.-W.; Hirosawa, T.; Tsuneda, T.; Hirao, K. Long-range corrected density functional calculations of chemical reactions: Redetermination of parameter. J. Chem. Phys. 2007, 126, 154105. 


\section{S2: Pump-Probe spectroscopy by repetition-rate tuning}

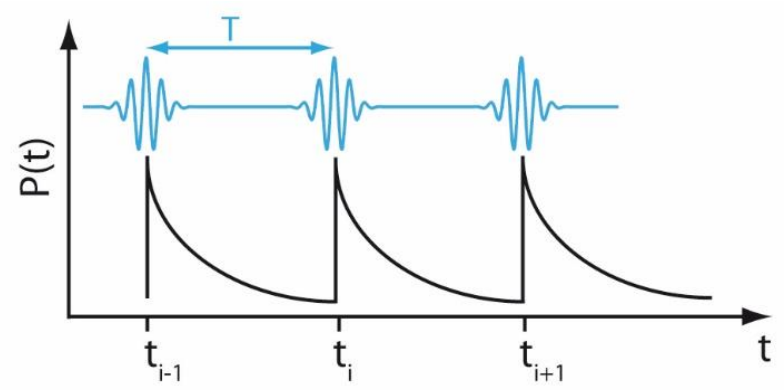

Figure S2. Scheme of the pump-probe setup via variation of the repetition rate (1/T).

To investigate the lifetime of the occupied triplet state we use a special form of pump-probe spectroscopy where we tune the repetition rate of the laser by a cavity dumper, which leads to a constant time delay $T$ between all pulses. In contrast to the conventional pump-probe scheme the state is not necessarily allowed to recover after each pulse, hence the known exponential decay law for the population $P(T)=e^{-T / \tau}$ is not valid. In the following we consider the occupation $P\left(t_{i}-\varepsilon\right)$ of the state just before the next pulse arrives and the corresponding occupation $P\left(t_{i}+\varepsilon\right)$ just after the pulse:

$$
P\left(t_{i}-\varepsilon\right)=P\left(t_{i-1}+\varepsilon\right) e^{-T / \tau}
$$

On the other hand the population $P\left(t_{i}+\varepsilon\right)$ just after the pulse is the sum of $P\left(t_{i}-\varepsilon\right)$ and a portion $\left(1-P\left(t_{i}-\varepsilon\right)\right) \cdot r$ which describes the ratio which can be excited by the next pulse:

$$
\begin{gathered}
P\left(t_{i}+\varepsilon\right)=P\left(t_{i}-\varepsilon\right)+\left(1-P\left(t_{i}-\varepsilon\right)\right) \cdot r \\
=P\left(t_{i}-\varepsilon\right)(1-r)+r
\end{gathered}
$$

Here $r$ denotes the population probability of the short-lived molecular state just before each pulse times the branching ratio for relaxation into the long-lived state. In the quasi-static case $P\left(t_{i}+\right.$ $\varepsilon)=P\left(t_{i-1}+\varepsilon\right)$, hence Eq. S2.1 and S2.2 lead to:

$$
P\left(t_{i}-\varepsilon\right)=\left[P\left(t_{i}-\varepsilon\right)(1-r)+r\right] e^{-T / \tau}
$$




$$
P(T)=\frac{r}{r-1+e^{T / \tau}}
$$

Eq. S2.4

In the limit of $\mathrm{T} \gg \tau$ the conventional pump-probe case is reproduced. 